Document downloaded from:

http://hdl.handle.net/10251/151278

This paper must be cited as:

Villalonga, R.; Díez, P.; Sánchez, A.; Aznar, E.; Martínez-Máñez, R.; Pingarrón, J. (2013). Enzyme-controlled sensing-actuating nanomachine based on Janus Au-mesoporous silica nanoparticles. Chemistry - A European Journal. 19(24):7889-7894. https://doi.org/10.1002/chem.201300723

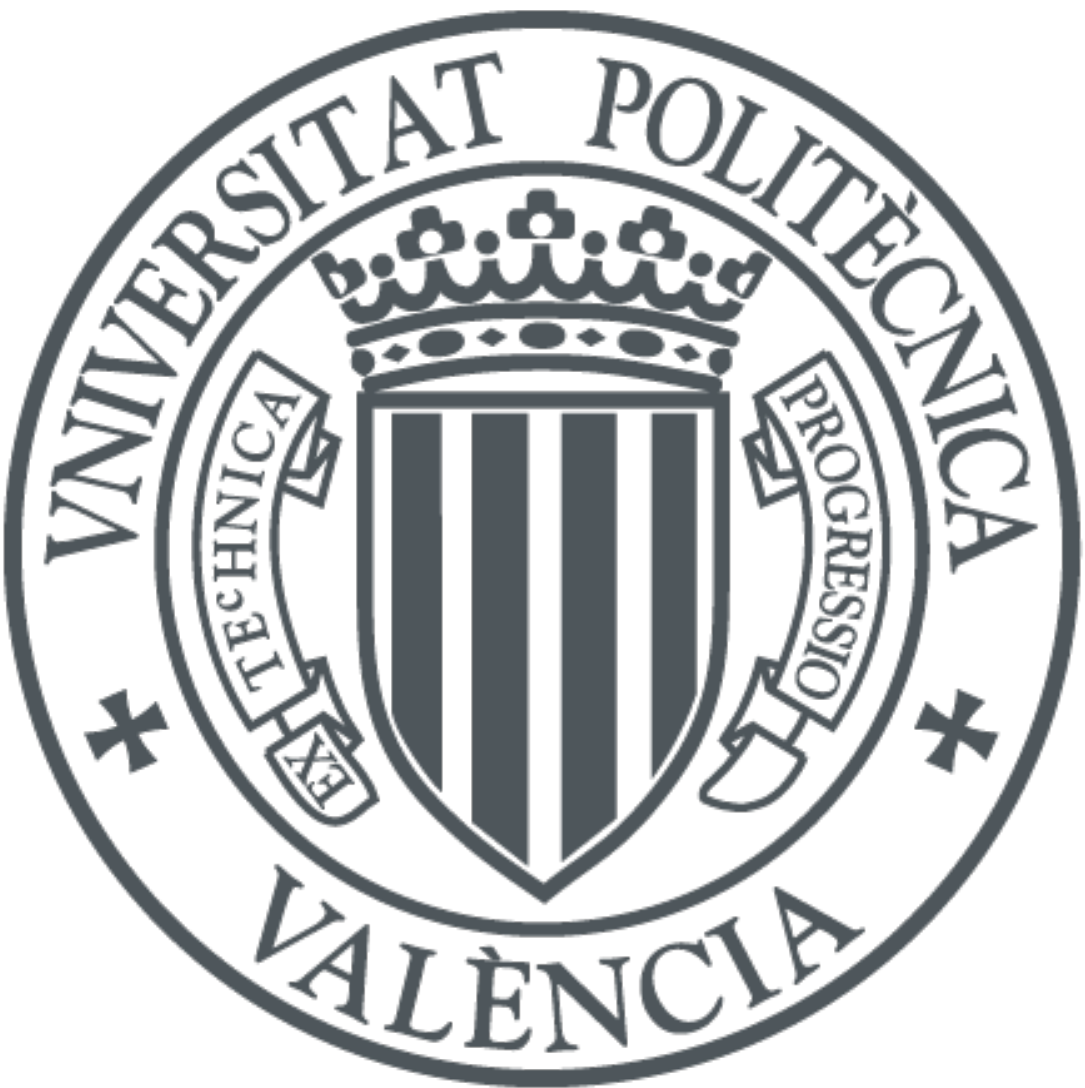

The final publication is available at

https://doi.org/10.1002/chem.201300723

Copyright John Wiley \& Sons

Additional Information 


\title{
Enzyme-controlled sensing-actuating nanomachine based on Janus Au-mesoporous silica nanoparticles
}

\author{
Reynaldo Villalonga, ${ }^{*[a, b]}$ Paula Díez, ${ }^{[a]}$ Alfredo Sánchez, ${ }^{[a]}$ Elena Aznar, ${ }^{[c, d]}$ Ramón Martínez- \\ Máñez, ${ }^{[c, d]}$ José M. Pingarrón*[a,b]
}

\author{
Dedication ((optional))
}

\begin{abstract}
Novel Janus nanoparticles with Au and mesoporous silica opposite faces were prepared by Pickering emulsion template using paraffin wax as oil phase. These anisotropic colloids were employed to design an integrated sensing-actuating nanomachine for the enzyme-controlled stimulus-responsible
\end{abstract}

delivery of a cargo compound. As a proof-of-concept, we demonstrated the successful use of the Janus colloids for controlled cargo delivery from the mesoporous silica face grafted with $\mathrm{pH}$ sensitive gate-like scaffoldings. The release of the cargo (tris(2,2'bipyridyl)ruthenium(II) chloride in this case) was mediated by the on-demand catalytic decomposition of urea by urease, which was covalently immobilized on the Au face.

Keywords: Janus nanoparticle, mesoporous silica, molecular gates, controlled release, enzyme

\section{Introduction}

The development of novel biologically-inspired nanomachines and smart drug delivery systems is linked to the tailor-made design of advanced nanomaterials with desired physical properties and chemical functionalities. ${ }^{[1]}$ Among these, particular interest has been devoted to the preparation of anisotropic colloidal particles which exhibit two surfaces of different chemical composition. ${ }^{[2]}$ These socalled "Janus nanoparticles" can be designed to show amphiphilic character as well as anisotropic electrical, magnetic or optical properties. ${ }^{[3]}$ Additionally, each face of the Janus nanoparticles can

[a] R. Villalonga, P. Díez, A. Sánchez, J.M. Pingarrón Department of Analytical Chemistry, Complutense University of Madrid 28040-Madrid, Spain , Fax: (+34) 913944329

E-mail: rvillalonga@quim.ucm.es, pingarro@quim.ucm.es

[b] IMDEA Nanoscience, Cantoblanco Universitary City, 28049-Madrid, Spain.

[c] E. Aznar, R. Martínez-Máñez Departamento de Química and CIBER de Bioingeniería, Biomateriales y Nanomedicina (CIBER-BBN), Universidad Politécnica de Valencia, Camino de Vera s/n, E-46022, Valencia, Spain , Fax: (+34) 963879349 E-mail: elazgi@upvnet.upv.es,rmaez@qim.upv.es

[d] Instituto de Reconocimiento Molecular y Desarrollo Tecnológico (IDM), Centro Mixto Universidad Politécnica de Valencia-Universidad de Valencia, Spain

Supporting information for this article is available on the WWW under http://www.chemeurj.org/ or from the author. be independently modified with selected ligands allowing specific functionalization with proteins and other biomacromolecules. ${ }^{[4]}$ These unique characteristics have favored the successful use of Janus nanomaterials as emulsion stabilizers, hydrophobic coat for textiles, self-propelled machines, imaging probes, and drug delivery systems. $^{[5]}$

On a different approach nanotechnology has proved to bring new innovative concepts to drug-delivery therapies. Drug delivery systems able to release active molecules to certain cells in a controlled manner have recently gained much attention. Among several potential drug delivery systems, mesoporous silica nanoparticles (MS) have been widely used in the past years as reservoirs for drug storage due to their unique properties such as a large specific volume, large loading capacity, low toxicity and easy functionalization. ${ }^{[6]}$ Moreover MS nanoparticles can be functionalized with molecular/supramolecular ensembles on their external surface to develop gated-MS showing "zero delivery" and capable to release of their cargo in response to external stimuli. Using this concept, MS displaying controlled release using several stimuli such as $\mathrm{pH}$, light, redox substances, small molecules and biomolecules have been reported. ${ }^{[7]}$ However, a potential limitation in these systems is related with the fact that the delivery MS-based support and the effector (i.e. agent that mediates the delivery) are not in the same nanoparticle and usually the gated materials are placed in a solution in which the triggering stimuli is applied (for instance light) or it is present in the media (for instance enzymes that induced the degradation of a specific gating coating).

As an advance in the design of more sophisticated nanoparticles for delivery applications we envisioned that it might possible to project systems in which the gating systems and an effector molecule could be placed in the same nano-device. In order to achieve this goal the strategy we have followed in shown in 
Scheme 1 and it involves the preparation of new Janus Au-MS nanoparticles by Pickering emulsion template using paraffin wax as oil phase. This allows obtaining two different surfaces with welldefined functionalization chemistries for the independent anchoring of the gated ensemble (on the MS face) and the effector molecule (on the Au face).

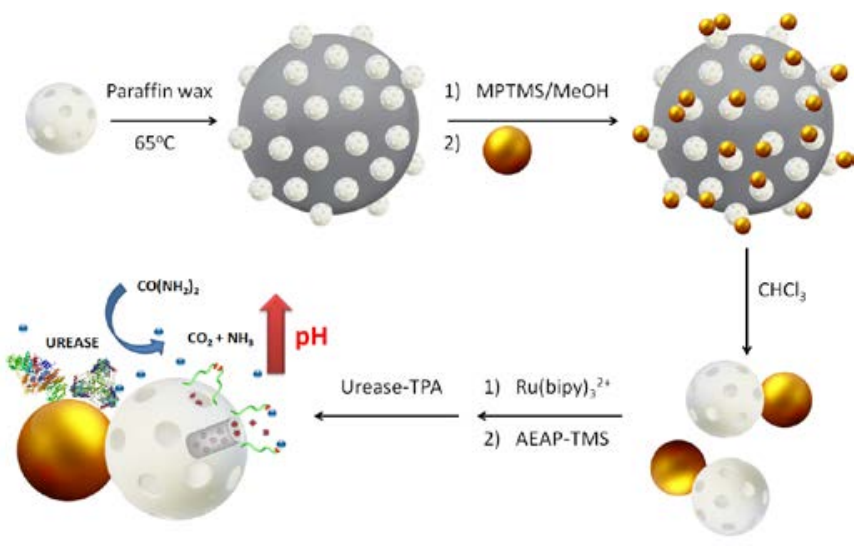

Scheme 1. Preparation of Janus Au-MS nanoparticles for enzyme-controlled release.

In this particular case, and as a first proof-of-concept, the MS part of the anisotropic colloid was capped with a $\mathrm{pH}$-responsive gate, whereas the gold surface was functionalized with the enzyme urease (EC 3.5.1.5). We reasoned that the gated mesoporous nano-devices would show "zero-release", yet selectively will open the $\mathrm{pH}$ responsive gate releasing the cargo in the presence of urea via urease-mediated urea hydrolysis which will lead to an increase of the $\mathrm{pH}$.

\section{Results and Discussion}

The starting MS nanoparticles (a calcined MCM41-like solid) were synthesized by alkaline hydrolysis of tetraethyl orthosilicate as inorganic precursor in the presence of the cationic surfactant cetyltrimethylammonium bromide as porogen species. ${ }^{[8]}$ The MS showed an average particle diameter of $97 \pm 15 \mathrm{~nm}$ and an MCM41 type channel-like mesoporous structure (Figure 1S in Supporting Information).

To synthesize the Janus nanoparticles, a rational design based on the manipulation of the Au-ligan-MS interface through maskprotecting assisted site-selective modification was employed. First, the surface of MS nanoparticles was partially masked by confining at the interface of Pickering emulsion. The exposed nanoparticle surface was further modified with a thiolated silane derivative, providing reactive sulfhydryl groups on this face. Au nanoparticles were then attached to the thiol-enriched face of the adsorbed MS nanoparticles through chemisorption reactions, forming stable anisotropic colloids. Several anisotropic nanomaterials have been previously prepared in excellent yield by manipulation of nanoparticle-ligand-nanoparticle interfaces in solutions. ${ }^{[9]}$ In the present work, such toposelective manipulation was performed in a solid-liquid interface.

According to this synthetic scheme, the as-prepared MS were first adsorbed onto the liquid-liquid interface of an emulsion prepared with water and molten paraffin wax, forming colloidosomes that remained stable after cooling. ${ }^{[10]}$ Although the formation of such colloidosomes was previously reported using silica nanoparticles with average diameters larger than $400 \mathrm{~nm},{ }^{[10,11]}$ we have proved here that this procedure can also be also used using MS nanoparticles of smaller diameter.
The toposelective modification of the MS nanoparticles adsorbed onto the colloidosome surface was performed by reaction with (3-mercaptopropyl)trimethoxysilane in water:methanol solution. After exhaustive washing, the colloidosomes containing the thiolfunctionalized MS nanoparticles were stirred in Au nanoparticles solution. Janus Au-MS nanoparticles were finally obtained with acceptable yield (about $85 \%$ for $\mathrm{J} 2$ sample) after dissolving the paraffin wax in $\mathrm{CHCl}_{3}$.
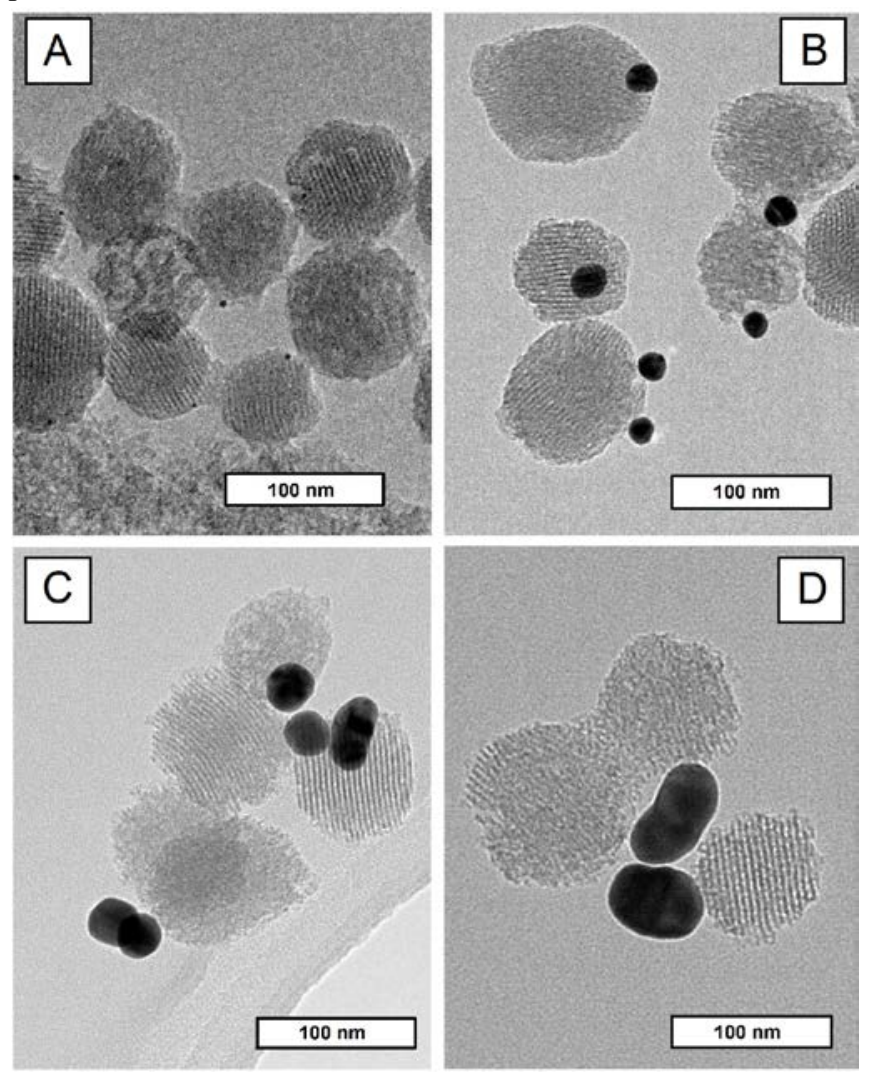

Figure 1. TEM images of J1 (A), J2 (B), J3 (C) and J4 (D) Janus Au-MS nanoparticles.

Figure 1 shows the TEM images of Janus colloids prepared by using Au nanoparticles of different sizes: $3.7 \pm 0.8 \mathrm{~nm}(\mathrm{~J} 1),{ }^{[12]} 20 \pm$ $2 \mathrm{~nm}(\mathrm{~J} 2), 31 \pm 6 \mathrm{~nm}(\mathrm{~J} 3)$ and $43 \pm 5 \mathrm{~nm}(\mathrm{~J} 4) .^{[13]}$ In all cases, anisotropic colloids were successfully synthesized, mainly with a 1:1 Au:MS nanoparticles ratio except for J1 samples in which the use of smaller Au nanospheres favored the attachment of more than one metal nanoparticle to the same MS colloid. J1 nanoparticles showed similar average diameter $(98 \pm 17 \mathrm{~nm})$ and size distribution than the native MS colloid, with only slight difference at higher values of diameter (Figure 2S in Supporting Information). This fact can be justified by the small diameter and low polydispersity of the attached Au nanoparticles. Moreover, J1 anisotropic colloids showed low metal content, which should be counterproductive for the further immobilization of urease and the preparation of the enzyme-controlled nanomachines.

Large average diameter and broad size distribution was observed for J3 (117 $\pm 19 \mathrm{~nm})$ and $\mathrm{J} 4(126 \pm 23 \mathrm{~nm})$ nanoparticles. This could be ascribed to the large diameter and size dispersion of $\mathrm{Au}$ nanoparticles prepared by Frens method using low citrate concentration, ${ }^{[13]}$ which often yield nanoparticles with non-spherical shape. In J4 samples, prepared using large Au nanoparticles, ${ }^{[13]}$ it was observed that some Au nanoparticles were assembled to more than one MS nanoparticle, yielding aggregated structures. This fact could be justified by the close packing of the MS nanoparticles onto the colloidosome surface, allowing the interaction of several thiolmodified mesoporous colloids with the same large and non-spherical 
Au nanoparticle. It should be highlighted that sample J2 (104 \pm 17 $\mathrm{nm}$ ) showed narrow distribution of size and maximum yield of AuMS anisotropic nanoparticles with 1:1 ratio, and accordingly this Janus colloid was selected for further experiments. A representative TEM image corresponding to J2 nanoparticles is shown in Figure 2. Other different TEM images of J2 nanoparticles are also shown in Figure 3S in Supporting Information.

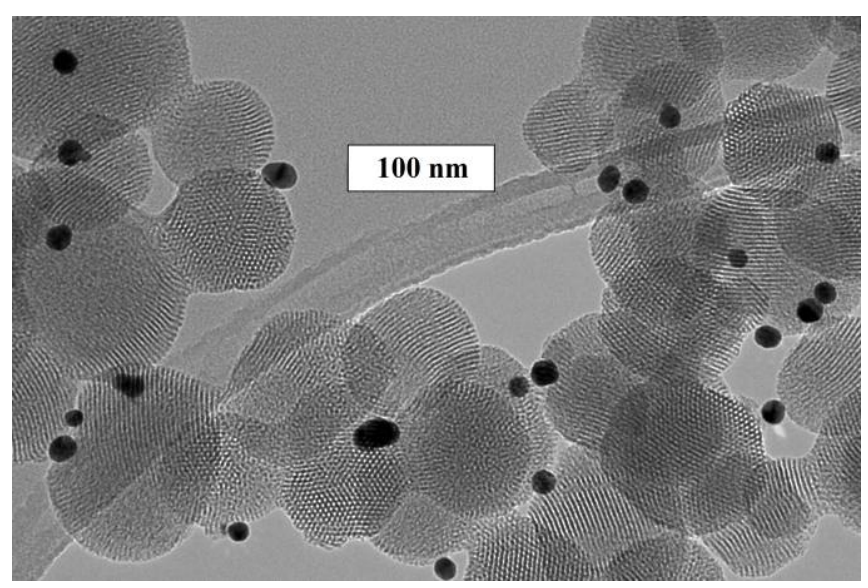

Figure 2. Representative TEM image of J2 nanoparticles.
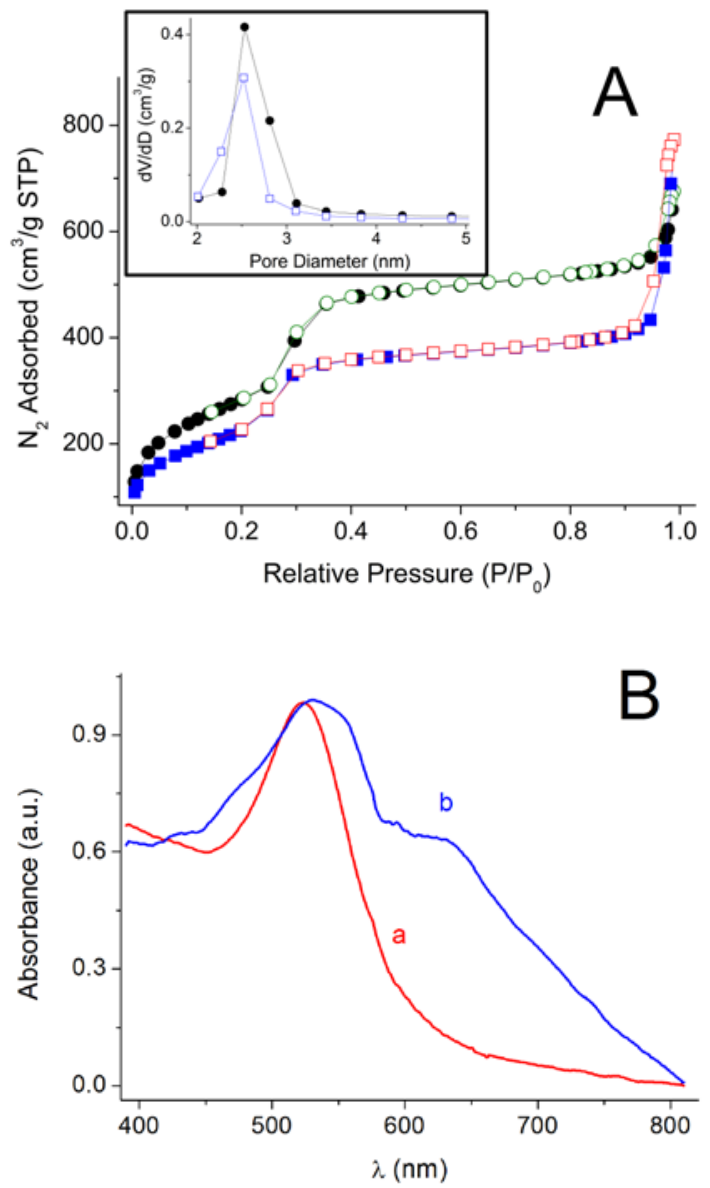

Figure 3. A) Nitrogen adsorption (closed)/desorption (open) isotherms for MS $(\bullet, \circ)$ and J2 nanoparticles $(\bullet, \square)$. Inset: pore size distribution of MS $(\bullet)$ and J2 ( $\square$ ) nanoparticles. B) Normalized visible spectra of Au (a) and J2 (b) nanoparticles.

The pore morphologies of the MS and Janus Au-MS nanoparticles in the $\mathrm{J} 2$ nanoparticles were determined by nitrogen adsorption/desorption surface analysis (BET isotherms and $\mathrm{BJH}$ pore size distributions). Figure 3A illustrates the corresponding nitrogen adsorption/desorption isotherms and the pore size distributions for the starting MS material and for the corresponding J2 Janus nanoparticles. Both nanomaterials showed type IV isotherms typical of mesoporous supports.

The absence of hysteresis loops for MS nanoparticles suggested that all pores are highly accessible. On the contrary, the small hysteresis loops observed at high relative pressure values in the Janus nanoparticle isotherm suggested that some pores were partially blocked most likely due to the toposelective silanization with (3-mercaptopropyl)trimethoxysilane and the attachment of the Au nanoparticles. In fact the attachment of Au nanoparticles on one face of the siliceous matrix reduced the BET specific surface area from $1037 \mathrm{~m}^{2} / \mathrm{g}$ in the starting MS to $820 \mathrm{~m}^{2} / \mathrm{g}$ in $\mathrm{J} 2$. Yet the average pore size of the MS support (ca. $2.5 \mathrm{~nm}$ ) was unchanged after formation of the anisotropic colloid (see inset of Figure 3A).

$\mathrm{UV}$-vis measurements in aqueous solutions were performed to provide insight into the surface characteristics of the Janus nanoparticles (Figure 3B). The starting Au colloids showed a single absorption band at $522 \mathrm{~nm}$, distinctive of the surface plasmon resonance of spherically-shaped nanospheres with about $20 \mathrm{~nm}$ diameter, whereas the attachment of the Au nanoparticles to the thiol-modified MS nanoparticles leads to a broadening and red-shift of the plasmon band in the $\mathrm{J} 2$ spectra. In addition, a broad shoulder band from $585 \mathrm{~nm}$ to $630 \mathrm{~nm}$ was also observed in the spectrum of $\mathrm{J} 2$ which can be tentatively attributed to the attachment of more than one Au nanospheres on the surface of the MS nanoparticles in some Janus particles, causing a coupling of the plasma modes due to metal particle-particle interactions. ${ }^{[14]}$
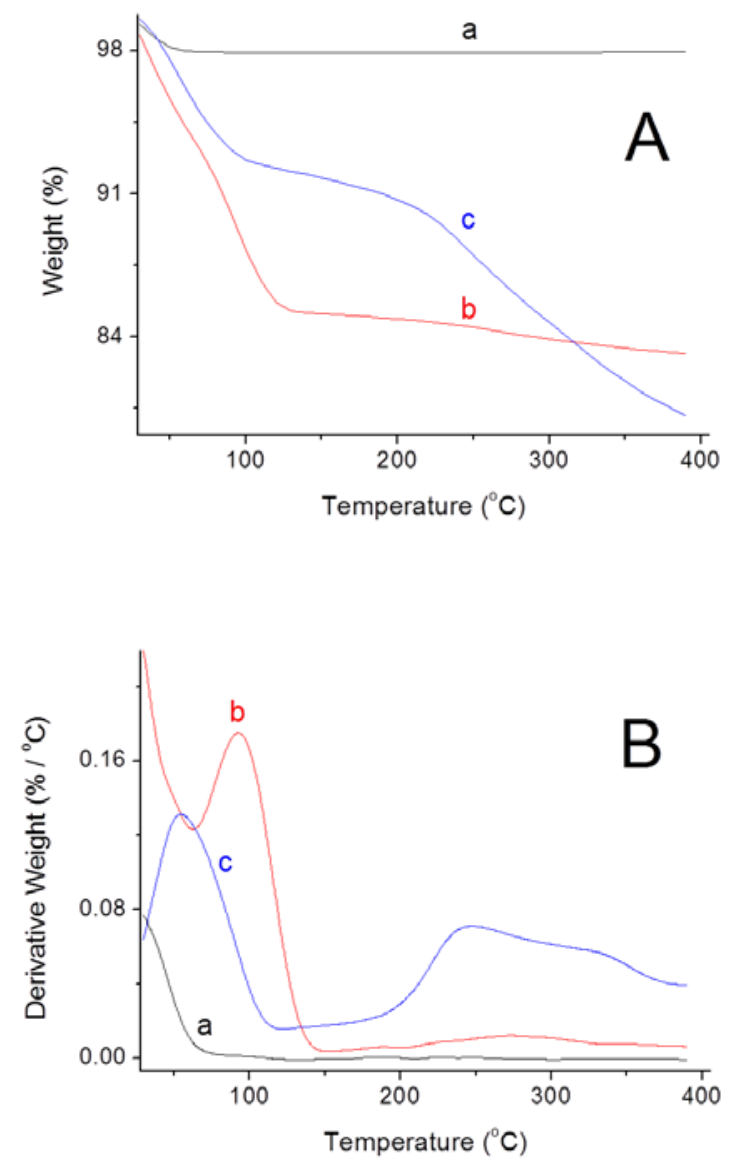

Figure 4. A) TG and B) DTG analysis for MS (a), J2 (b) and J2Ru (c) nanoparticles. 
J2 colloid was further employed as nanosized hardware for the assembly of a self-controlled nanomachine able to release a cargo compound by an enzyme-based $\mathrm{pH}$-mediated mechanism. In a first step J2 nanoparticles were loaded with tris(2,2' bipyridyl)ruthenium(II) chloride ([Ru(bpy) $\left.]_{3}\right]^{2+}$ ) as model dye for monitoring cargo delivery. ${ }^{[\mathrm{c}]}$ Thereafter, an excess of the alkyl amino derivative 3-(2-aminoethylamino)propyltrimethoxysilane (the $\mathrm{pH}$-responsive molecular gate) was anchored on the external surface of the mesoporous face to yield the J2Ru nanomaterial. ${ }^{[7]}$ It should be mentioned that Janus nanoparticles exhibited good water solubility, but its stability in aqueous solutions was reduced after modification with 3-(2-aminoethylamino)propyltrimethoxysilane.

Thermal analysis of the MS, J2 and J2Ru nanomaterials was accomplished, the TG/DTG curves being displayed in Figures 4 (A,B). The unmodified MS nanoparticles exhibited a slight weight loss at temperatures up to ca. $70^{\circ} \mathrm{C}$, which was attributed to the thermodesorption of physically adsorbed water molecules from the silica surface. At higher temperatures no weight loss was apparent showing relatively flat TG/DTG curves. ${ }^{[15]}$ Janus nanoparticles showed a more noticeable weight loss at temperatures up to ca. $140^{\circ} \mathrm{C}$ suggesting that higher amount of water molecules were adsorbed on the Au nanoparticles surface. The anisotropic nanoparticle exhibited a second thermal-induced transformation, with maximum rate of weight loss at approximately $275^{\circ} \mathrm{C}$, which could be associated with the decomposition of the bonded thiol ligands but also with the condensation of the ligand's side silanols with one another or with surface silanols.

In comparison with $\mathrm{J} 2$, the weight loss profile for $\mathrm{J} 2 \mathrm{Ru}$ exhibited a lower decrease at $\mathrm{T}<120^{\circ} \mathrm{C}$, suggesting lower amount of water molecules physically adsorbed on this nanomaterial. This fact could be justified by the azeotropic treatment of the Janus nanoparticles before adsorption of the $\left[\mathrm{Ru}(\mathrm{bpy})_{3}\right]^{2+}$ complex and silanization with 3-(2-aminoethylamino)propyltrimethoxysilane, as well as to the introduction of the long chain aminosilane groups on the MS face. The weight loss at higher temperatures can be divided into two regions. The first region, with maximum rate of weight loss at $245^{\circ} \mathrm{C}$, could be attributed to decomposition/condensation of the bounded ligands. The second thermal-induced process, which showed a maximum rate of transformation at about $330^{\circ} \mathrm{C}$, could be associated with the decomposition of the $\left[\mathrm{Ru}(\mathrm{bpy})_{3}\right]^{2+}$ complex, which is thermally stable up to $320{ }^{\circ} \mathrm{C}^{[16]}$ The loading of the [Ru(bpy) $\left.)_{3}\right] \mathrm{Cl}_{2}$ dye in the J2Ru material amounted to $62 \mathrm{mg} / \mathrm{g} \mathrm{J} 2 \mathrm{Ru}$ in weight, as determined by the TG curve. Moreover the content of the anchored amine-based gate amounted to $49 \mathrm{mg} / \mathrm{g} \mathrm{J} 2 \mathrm{Ru}$ in weight.

Figure 5A shows an FT-IR spectrum of the MS, J2 and J2Ru nanoparticles. MS nanoparticles showed the characteristics IR absorption bands of siliceous materials at $456 \mathrm{~cm}^{-1}$ attributed to the vibration of the Si-O bonds, a shoulder at $576 \mathrm{~cm}^{-1}$ ascribed to cyclic Si-O-Si structures, at $803 \mathrm{~cm}^{-1}$ attributed to $\mathrm{SiO}_{4}$ tetrahedrons, at $946 \mathrm{~cm}^{-1}$ attributed to the $\mathrm{Si}-\mathrm{OH}$ groups, and a band at $1080 \mathrm{~cm}^{-1}$ with a shoulder at $1200 \mathrm{~cm}^{-1}$ ascribed to the bond stretching vibrations of Si-O-Si. ${ }^{[17]}$ The broad band at $3700-3000 \mathrm{~cm}^{-1}$ can be ascribed to the $\mathrm{O}-\mathrm{H}$ bonding vibration of adsorbed water and $\mathrm{SiO}-\mathrm{H}$ groups, and the band at $1629 \mathrm{~cm}^{-1}$ is attributed to the deformation vibration of the $\mathrm{HO}-\mathrm{H}$ bond in water molecules.

Spectrum of J2 nanoparticles presented the antisymmetric and symmetric stretching vibrations of the $\mathrm{CH}_{2}$ groups at $2933 \mathrm{~cm}^{-1}$ and $2856 \mathrm{~cm}^{-1}$, confirming the modification of the MS nanomaterial with (3-mercaptopropyl)trimethoxysilane. The absence of the characteristic bands of S-H vibration in the range of $2500-2600 \mathrm{~cm}^{-1}$ suggested that the thiol groups were chemisorpted to the $\mathrm{Au}$ nanoparticle surface. Loading of $\mathrm{J} 2$ with $\left[\mathrm{Ru}(\mathrm{bpy})_{3}\right]^{2+}$ and derivatization with 3-(2-aminoethylamino)propyltrimethoxysilane was further confirmed by the bands at $2946 \mathrm{~cm}^{-1}$ and $2859 \mathrm{~cm}^{-1}$ (U[C$\mathrm{H}]), 1633 \mathrm{~cm}^{-1}\left(\delta_{[\mathrm{N}-\mathrm{H}]}\right), 1568 \mathrm{~cm}^{-1}\left(\mathrm{U}_{[\mathrm{C}-\mathrm{C}]}+\mathrm{U}_{[\mathrm{C}=\mathrm{C}-\mathrm{H}]}\right), 1480 \mathrm{~cm}^{-1}$ (U[C-C] $+\mathrm{v}[\mathrm{C}=\mathrm{C}-\mathrm{H}]$ ) and $1319 \mathrm{~cm}^{-1}$ ( $\left[\mathrm{N}_{\mathrm{NC}-\mathrm{H}]}\right)$ in the J2Ru spectrum. ${ }^{[18]}$ Another important characteristic is the drop in intensity of the band around $950 \mathrm{~cm}^{-1}$, which is attributed to the vibration of the silanol groups, confirming the modification of the mesoporous silica surface with the amine-bearing silane. ${ }^{[7]}$
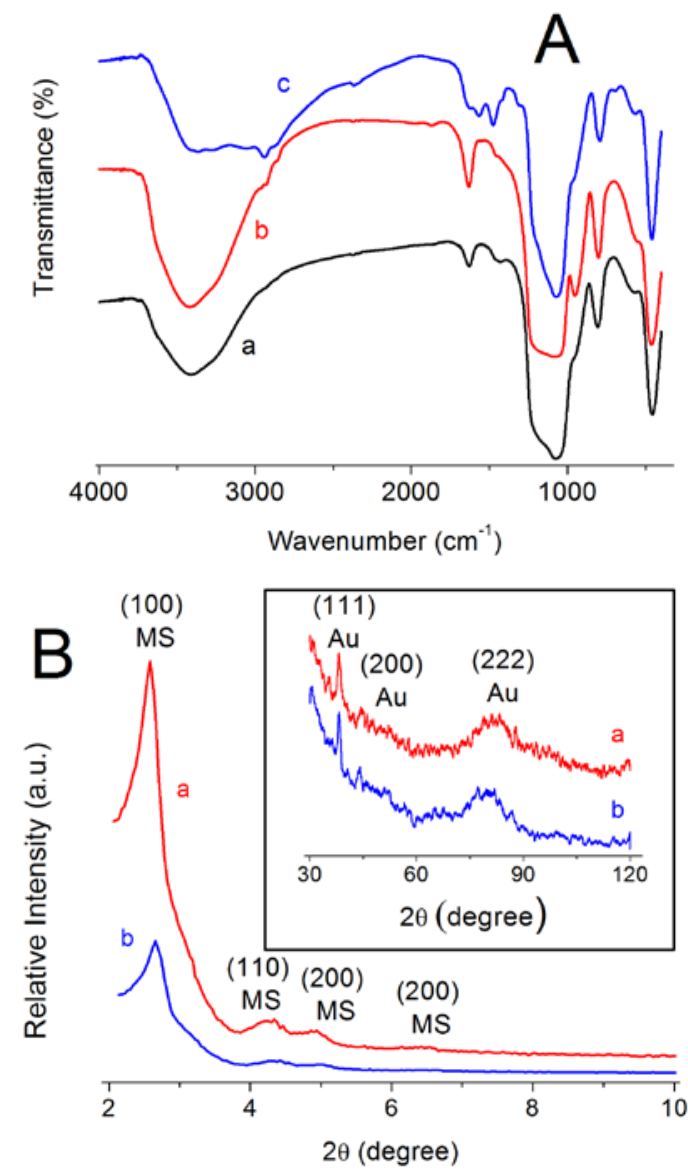

Figure 5. A) FT-IR analysis for MS (a), J2 (b) and J2Ru (c) nanoparticles. B) X-ray diffraction of J2 (a) and J2Ru (b) nanoparticles.

The powder X-ray diffraction patterns of J2 and J2Ru are shown in Figure 5B. The low angle diffractogram of $\mathrm{J} 2$ shows four distinguishable peaks at $2.59^{\circ}, 4.30^{\circ}, 4.93^{\circ}$ and $6.46^{\circ}$ which correspond to (100), (110), (200) and (210) of MCM-41 with dspacing values of $3.40 \mathrm{~nm}, 2.05 \mathrm{~nm}, 1.79 \mathrm{~nm}$ and $1.37 \mathrm{~nm}$, respectively. This pattern suggested perfect long-range order in this mesoporous nanomaterial. Additionally, from these data and the pore value diameter obtained from nitrogen adsorption isotherms, an ao cell parameter of $3.93 \mathrm{~nm}$ and a pore wall thickness of $1.40 \mathrm{~nm}$ can be calculated. Moreover, the diffraction pattern of $\mathrm{J} 2$ at high angle showed three peaks at $38.34^{\circ}, 44.50^{\circ}$ and $81.21^{\circ}$, corresponding to the (111), (200) and (222) Bragg reflections for cubic gold nanocrystals, ${ }^{[19]}$ confirming the Janus architecture previously observed by TEM. J2Ru sample showed similar X-ray diffraction pattern, suggesting that the loading process with the dye and the further functionalization with amine groups did not damage neither the mesoporous MCM-41 type structure nor of the gold face of the Janus colloid. ${ }^{[7]}$

In order to prepare the self-controlled enzyme-powered nanodevice for cargo delivery, the $\mathrm{Au}$ face of the Jan2Ru nanoparticles were functionalized with the enzyme urease. For 
preparing an enzyme form suitable to be anchored on the metal surface, urease was covalently modified with 3,3'dithiobis(sulfosuccinimidylpropionate) (DTSSP). After reduction of the dithiol linkage with $\mathrm{NaBH}_{4}$, the modified enzyme solution was dialyzed and finally incubated with J2Ru to yield the J2Ru-U nanomaterial. All these processes were performed in $50 \mathrm{mM}$ sodium phosphate buffer, $\mathrm{pH} 7.0$, at $4^{\circ} \mathrm{C}$.

The ability of J2Ru-U nanoparticle to deliver the $\left[\mathrm{Ru}(\mathrm{bpy})_{3}\right]^{2+}$ dye under urease control was tested in $5 \mathrm{mM}$ sodium acetate buffer at $\mathrm{pH} 4.0$ and 5.0. It is well know that the $\mathrm{pH}$-activity profile of urease has a bell-shaped behavior with maximum at $\mathrm{pH} 7.0 .^{[20]}$ However, more than $66 \%$ of the 3 -(2-aminoethylamino) propyltrimethoxysilane grafting moieties on the MS surface are unprotonated at this $\mathrm{pH}$ value, ${ }^{[7 \mathrm{a}]}$ then the gate-like ensembles was partially open. To avoid this undesirable effect, we used buffer solution of $\mathrm{pH} 5.0$ where urease retains about $60 \%$ of its maximum catalytic activity, ${ }^{[20]}$ and not appreciable release of the dye from $\mathrm{J} 2 \mathrm{Ru}-\mathrm{U}$ was experimentally observed in the control experiments.

In a typical release assay, $10 \mathrm{mg}$ of J2Ru-U were suspended in $10 \mathrm{~mL}$ of buffer solution containing $180 \mathrm{mM}$ urea, and shaken over time at $25^{\circ} \mathrm{C}$. Aliquots were taken at scheduled times, centrifuged and the absorbance was measured at $454 \mathrm{~nm}$. As control experiments, J2Ru-U samples were suspended in similar buffer solution without urea. In order to ensure saturating conditions for substrate in the enzyme catalyzed reaction, concentration of urea about 100-fold larger than $K_{M}=1.3 \mathrm{mM}$ was employed.

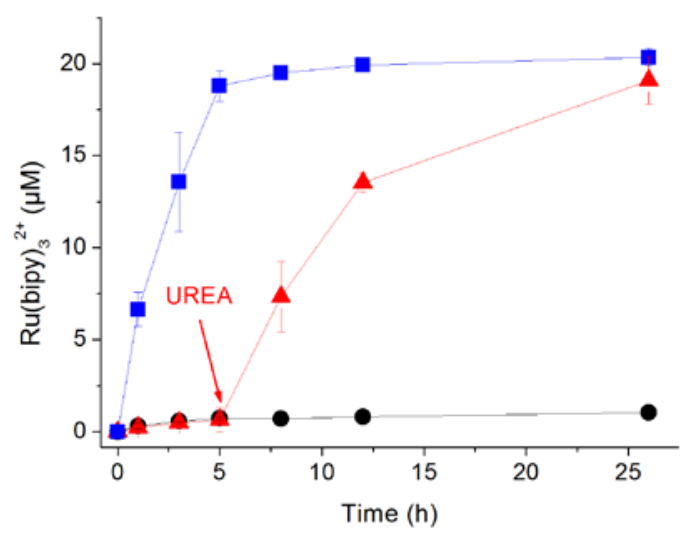

Figure 6. Kinetics of dye release from J2Ru-U in $5 \mathrm{mM}$ sodium acetate buffer $\mathrm{pH} 5.0$ in the absence $(\bullet)$ and the presence of $180 \mathrm{mM}$ urea at $\mathrm{t}=0(\boldsymbol{\bullet})$ or $\mathrm{t}=5 \mathrm{~h}(\boldsymbol{\Delta})$.

Figure 6 shows the time-course of $\left[\mathrm{Ru}(\mathrm{bpy})_{3}\right]^{2+}$ release from the pores of the J2Ru-U nanoparticles. The concentration of dye delivered to the solution increased progressively with the time of incubation for the mixture containing urea at $\mathrm{pH} 5.0$, reaching plateau value of about $19 \mu \mathrm{M}$ after $5 \mathrm{~h}$ of incubation. On the contrary, no significant increase in the absorbance measured at 454 nm was observed for control solution containing no urea.

Urease catalyzed the transformation of urea to $\mathrm{CO}_{2}$ and $\mathrm{NH}_{3}$ yielding a progressive increase in the $\mathrm{pH}$ value of the incubation solutions, as it was qualitatively demonstrated by adding bromothymol blue to control assays. As it was previously reported, the 3-(2-aminoethylamino)propyltrimethoxysilane moieties located on the MS surface became gradually deprotonated when the solution $\mathrm{pH}$ reached alkaline values. ${ }^{[7 \mathrm{a}]}$ Consequently, the nanoscopic molecular gates located at the pore outlets trended to be opened favoring the release of the dye.

To provide insight into this enzyme-controlled dye release mechanism, J2Ru-U nanomaterials were incubated in buffer solution without urea under the conditions described above. Urea (180 mM final solution) was then added after $5 \mathrm{~h}$ of incubation, the results being shown in Figure 6. No appreciable dye release was observed during the first $5 \mathrm{~h}$ of incubation but the further increase in the absorbance at $454 \mathrm{~nm}$ revealed that the dye was successfully delivered to the solution after adding urea.

\section{Conclusion}

In summary, we described the preparation of Janus-type nanoparticles having Au and MS opposite faces and have used them for the design of an integrated nano-device containing on the same nanoparticle a gating systems and an effector molecule for the stimulus-responsible delivery of a cargo. In this particular case, as a proof-of-concept the release was mediated by the on-demand catalytic decomposition of urea by the enzyme urease, which was immobilized on the Au face, whereas the gated ensemble consisted of a pH-responsive system which was anchored on the pore outlets of the MS phase. In spite of the many reports dealing with the preparation of silica-based anisotropic colloids, approaches to produce MS-based Janus nanoparticles and their evaluation as ondemand control release systems are scarce to our knowledge. We believe that the possibility of incorporate different effector molecules and gated ensembles on this Janus-type integrated nanoarchitecture, which constitutes a proof-of-concept, open new routes for the development of novel biologically-inspired smart nanomachines for drug delivery and sensing applications and research is this line is currently being performed by us.

\section{Experimental Section}

Preparation of MS nanoparticles: ${ }^{[8]}$ Cetyltrimethylammonium bromide $(3.0 \mathrm{~g})$ was dissolved in $1.44 \mathrm{~L}$ of water under sonication. $\mathrm{NaOH}$ solution $(2.0 \mathrm{~mol} / \mathrm{L}, 10.5 \mathrm{~mL})$ was then added and the temperature of the mixture was adjusted to $80^{\circ} \mathrm{C}$. Tetraethoxysilane $(15.0 \mathrm{~mL})$ was added dropwise to the surfactant solution within 5 min under vigorous magnetic stirring. The mixture was allowed to react for $2 \mathrm{~h}$. The resulting white solid was filtered, washed with water and methanol, and then dried in desiccator. The solid was finally calcinated at $550{ }^{\circ} \mathrm{C}$ for $5 \mathrm{~h}$.

Preparation of $3.7 \mathrm{~nm}$ Au nanoparticles: ${ }^{[12]}$ An ice-cold, freshly prepared $0.1 \mathrm{M} \mathrm{NaBH}_{4}$ solution $(600 \mu \mathrm{L})$ was quickly added to $20 \mathrm{~mL}$ aqueous solution containing $300 \mu \mathrm{M}$ $\mathrm{HAuCl}_{4}$ and $300 \mu \mathrm{M}$ trisodium citrate under continuous stirring. The mixture was stirred for $30 \mathrm{~min}$, and 100 -fold diluted with $300 \mu \mathrm{M}$ trisodium citrate before $\mathrm{J} 1$ preparation.

Preparation of $18 \mathrm{~nm}, 29 \mathrm{~nm}$ and $41 \mathrm{~nm}$ Au nanoparticles: ${ }^{[13]}$ Freshly prepared $3 \mu \mathrm{M}$ $\mathrm{HAuCl}_{4}$ solutions $(50 \mu \mathrm{L})$ were heated to boiling. Au nanoparticles of $18 \mathrm{~nm}, 29 \mathrm{~nm}$ and $41 \mathrm{~nm}$ were synthesized by adding $750 \mu \mathrm{L}, 500 \mu \mathrm{L}$ and $300 \mu \mathrm{L}$ of $3.9 \mu \mathrm{M}$ trisodium citrate solution, respectively. The mixtures were heated for $10 \mathrm{~min}$, cooled to room temperature and finally raised to $50 \mathrm{~mL}$ with ultrapure water.

Preparation of Janus Au-MS nanoparticles: Janus nanoparticles were synthesized by adapting two methods previously reported in literature. ${ }^{[10]}$ MS nanoparticles (200 mg) were dispersed homogeneously in $10 \mathrm{~mL}$ of $1.0 \mu \mathrm{M}$ of CTAB in $6.7 \%$ ethanol aqueous solution. The mixture was heated at $75^{\circ} \mathrm{C}$ and then $1 \mathrm{~g}$ of paraffin wax was added. When the paraffin wax was melted, the mixture was vigorously mixed at $25000 \mathrm{rpm}$ during 2 min using an Ultra-Turrax T-10 homogenizer (IKA, Germany). The resulting emulsion was further mixed during $5 \mathrm{~min}$ at $4000 \mathrm{rpm}$ and $75^{\circ} \mathrm{C}$, using the same apparatus. The resulting Pickering emulsion was then cooled to room temperature, mixed with $10 \mathrm{~mL}$ methanol and treated with $200 \mu \mathrm{L}$ of (3-mercaptopropyl)trimethoxysilane. After $3 \mathrm{~h}$ under magnetic stirring, the silanized emulsion was filtered, three-times washed with methanol and further dispersed in $400 \mathrm{~mL}$ of the corresponding $3 \mu \mathrm{M}$ Au nanoparticles aqueous solutions. The mixture was stirred overnight, then filtered and exhaustively washed with ultrapure water. The solid was suspended in ethanol, centrifuged and washed two-times with ethanol and three-times with chloroform. The Janus nanoparticles were finally dried and kept in dissecator until use.

Preparation of J2Ru-U: To synthesize J2Ru, $100 \mathrm{mg}$ of $\mathrm{J} 2$ and $60 \mathrm{mg}$ of tris(2,2'bipyridyl)ruthenium(II) chloride hexahydrate were suspended in $10 \mathrm{~mL}$ of anhydrous 
acetonitrile inside a round-bottom flask connected to a Dean-Stark trap under A atmosphere. ${ }^{[7 a]}$ The suspension was refluxed at $110{ }^{\circ} \mathrm{C}$ in azeotropic distillation, collecting about $4 \mathrm{~mL}$ in the trap in order to remove the adsorbed water. The mixture was stirred for $24 \mathrm{~h}$ at room temperature to load the dye into the MS face pores. Afterward, an excess of 3-(2-aminoethylamino)propyltrimethoxysilane $(500 \mu \mathrm{L})$ was added, and the suspension was stirred for $5.5 \mathrm{~h}$. Finally, the orange solid (J2Ru) was filtered off, washed two times with $30 \mathrm{~mL}$ of $\mathrm{CH}_{3} \mathrm{CN}$, and dried at $70{ }^{\circ} \mathrm{C}$ for $12 \mathrm{~h}$.

To synthesize J2Ru-U, $4.0 \mathrm{mg}$ of urease and $4.0 \mathrm{mg}$ of 3,3' dithiobis(sulfosuccinimidylpropionate) were dissolved in $5.0 \mathrm{~mL}$ of $50 \mathrm{mM}$ sodium phosphate buffer, $\mathrm{pH} 7.0$, and stirred during $2 \mathrm{~h}$ at $4{ }^{\circ} \mathrm{C}$. Afterward, $200 \mu \mathrm{L}$ of $100 \mathrm{mM}$ $\mathrm{NaBH}_{4}$ solution were added, and the mixture was stirred at $4{ }^{\circ} \mathrm{C}$ for $30 \mathrm{~min}$. The solution was exhaustively dialyzed vs. $50 \mathrm{mM}$ sodium phosphate buffer, $\mathrm{pH} 7.0$ using Amicon Ultra-05 centrifugal filter units with Ultracel-10 membranes (Millipore, USA), and finally concentrated to about $10 \mathrm{mg} / \mathrm{mL}$ concentration. The modified enzyme solution was then added to $50 \mathrm{~mL}$ sodium phosphate buffer, $\mathrm{pH} 7.0$, containing $50 \mathrm{mg}$ of J2Ru, and stirred at $4^{\circ} \mathrm{C}$ overnight. The resulting solid (J2Ru-U) was finally isolated by centrifugation, washed several times with sodium phosphate buffer, $\mathrm{pH}$ 7.0, dried and kept in refrigerator until use.

Characterization: Transmission electron microscopy (TEM) measurements were performed with JEOL JEM-3000 F and JEOL JEM-2100 microscopes. The morphology of the colloidosomes was characterized using high resolution field emission scanning electron microscopy (FE-SEM) with a JEOL JSM-6335F electron microscope (JEOL Ltd., Japan). FT-IR spectra were acquired with a Perkin-Elmer instrument. Spectrophotometric measurements were performed using an Agilent 8453 UV/VIS spectrophotometer (Hewlett Packard, USA). Power X-ray diffraction (XRD) was performed with an X'Pert MRD diffractometer (PANanalytical B.V., The Netherlands). Nitrogen adsorption/desorption isotherms and pore size distributions were determined with an ASAP 2020 Physisorption Analyzer (Micromeritics, USA). Thermal analysis was performed with a TA Instruments SDT-Q600 apparatus (USA). FT-IR spectra were acquired with a Nicolet Nexus 670/870 spectrometer (Thermo Fisher Scientific Inc., USA)

\section{Acknowledgements}

R. Villalonga acknowledges to Ramón \& Cajal contract from the Spanish Ministry of Science and Innovation. Financial support from the Spanish Ministry of Science and Innovation CTQ2011-24355, CTQ2009-12650, CTQ2009-09351, MAT2009-14564C04-01, MAT2012-38429-C04-01 and Comunidad de Madrid S2009/PPQ-1642, programme AVANSENS is gratefully acknowledged. The Generalitat Valencia (project PROMETEO/2009/016) is also acknowledged.

[1] N. Hasirci, in Nanomaterials and Nanosystems for Biomedical Applications (Ed.: M.R. Mozafari), Springer, Germany, 2007.

[2] a) A. Perro, S. Reculusa, S. Ravaine, E. Bourgeat-Lami, E. Duguet, J. Mater Chem. 2005, 15, 3745; b) S. Jiang, Q. Chen, M. Tripathy, E. Luijten, K.S. Schweizer, S. Granick, Adv. Mater. 2010, 22, 1060.

[3] M. Lattuada, T.A. Hatton, Nano Today 2011, 6, 286.

[4] J.L. Tang, K. Schoenwald, D. Potter, D. White, T. Sulchek, Langmuir 2012, 28 , 10033
[5] a) J.W. Kim, D. Lee, H.C. Shum, D.A. Weitz, Adv. Mater. 2008, 20, 3239; b) A Synytska, R. Khanum, L. Ionov, C. Cherif, C. Bellmann, ACS Appl. Mater. Interfaces 2011, 3, 1216; c) J.R. Howse, R.A. Jones, A.J. Ryan, T. Gough, R. Vafabakhsh, R. Golestanian, Phys. Rev. Lett. 2007, 99, 048102; d) M. Yoshida, K.H. Roh, J. Lahann, Biomaterials 2007, 28, 2446; e) A.K. Salem, P.C. Searson, K.W. Leong, Nat. Mater. 2003, 2, 668.

[6] a) L. Zhang, F. Zhang, W.F. Dong, J.F. Song, Q.S. Huo, H.B. Sun, Chem Commun. 2011, 47, 1225; b) J.E. Lee, N. Lee, T. Kim, J. Kim, T. Hyeon, Acc. Chem. Res. 2011, 44, 893.

[7] a) R. Casasús, E. Climent, M.D. Marcos, R. Martínez-Máñez, F. Sancenón, J. Soto, P. Amorós, J. Cano, E. Ruiz, Am. Chem. Soc. 2008, 130, 1903; b) A Bernardos, L. Mondragón, E. Aznar, M.D. Marcos, R. Martínez-Máñez, F. Sancenón, J. Soto, J.M. Barat, E. Pérez-Payá, C. Guillem, P. Amorós, ACS Nano 2010, 4, 6353; c) I. Candel, A. Bernardos, E. Climent, M.D. Marcos, R. Martínez-Máñez, F. Sancenón, J. Soto, A. Costero, S. Gil, M. Parra, Chem. Commun. 2011, 47, 8313

[8] Y. Zhao, B.G. Trewyn, I.I. Slowing, V.S.Y. Lin, J. Am. Chem. Soc. 2009, 131, 8398

[9] Y. Feng, J. He, H. Wang, Y.Y. Tay, H. Sun, L. Zhu, H. Chen, J. Am. Chem. Soc 2012, 134, 2004; T. Chen, G. Chen, S. Xing, T. Wu, H. Chen, Chem. Mat. 2010, 22, 3826.

[10] L. Hong, S. Jiang, S. Granick, Langmuir 2006, 22, 9495; A. Perro, F. Meunier, V. Schmitt, S. Ravaine, Colloids Surf. A 2009, 332, 57.

[11] D. Rodríguez-Fernández, J. Pérez-Juste, I. Pastoriza-Santos, L.M. Liz-Marzán, ChemistryOpen 2012, 1, 90.

[12] N.R. Jana, L. Gearheart, C.J. Murphy, Langmuir 2001, 17, 6782.

[13] G. Frens, Nature 1973, 241, 20

[14] S.K. Ghosh, T. Pal, Chem. Rev. 2007, 107, 4797.

[15] a) C.P. Jaroniec, M. Kruk, M. Jaroniec, A. Sayari, J. Phys. Chem. B 1998, 102, 5503; b) C.P. Jaroniec, R.K. Gilpin, M. Jaroniec, J. Phys. Chem. B 1997, 101, 6861.

[16] P. Innocenzi, H. Kozuka, T. Yoko, J. Phys. Chem. B 1997, 101, 2285

[17] M. Stefanescu, M. Stoia, O. Stefanescu, J. Sol-Gel Sci. Techn. 2007, 41, 71

[18] M. Ammam, E.B. Easton, Sens. Actuators B 2012, 161, 520

[19] D.V. Leff, L. Brandt, J.R. Heath, Langmuir 1996, 12, 4723.

[20] B. Sahoo, S.K. Sahu, P. Pramanik, J. Mol. Catal. B: Enzym. 2011, 69, 95.

Received: ((will be filled in by the editorial staff))

Revised: ((will be filled in by the editorial staff)) Published online: ((will be filled in by the editorial staff)) 
Entry for the Table of Contents (Please choose one layout only)

\section{Layout 1:}

Janus nanoparticles based

nanomachine

Reynaldo Villalonga,* Paula Díez, Alfredo Sánchez, Elena Aznar,

Ramón Martínez-Máñez, José M.

Pingarrón*............. Page - Page

Enzyme-controlled sensing-

actuating nanomachine based on

Janus Au-mesoporous silica

nanoparticles
Integrated sensing-actuating nanomachine based on Janus Aumesoporous silica nanoparticles with gate-like scaffolding for enzymecontrolled cargo release 


\section{Supporting Information}

\section{Enzyme-controlled sensing-actuating nanomachine based on Janus Au-}

mesoporous silica nanoparticles

Reynaldo Villalonga, Paula Díez, Alfredo Sánchez, Elena Aznar, Ramón Martínez-Máñez, José M. Pingarrón
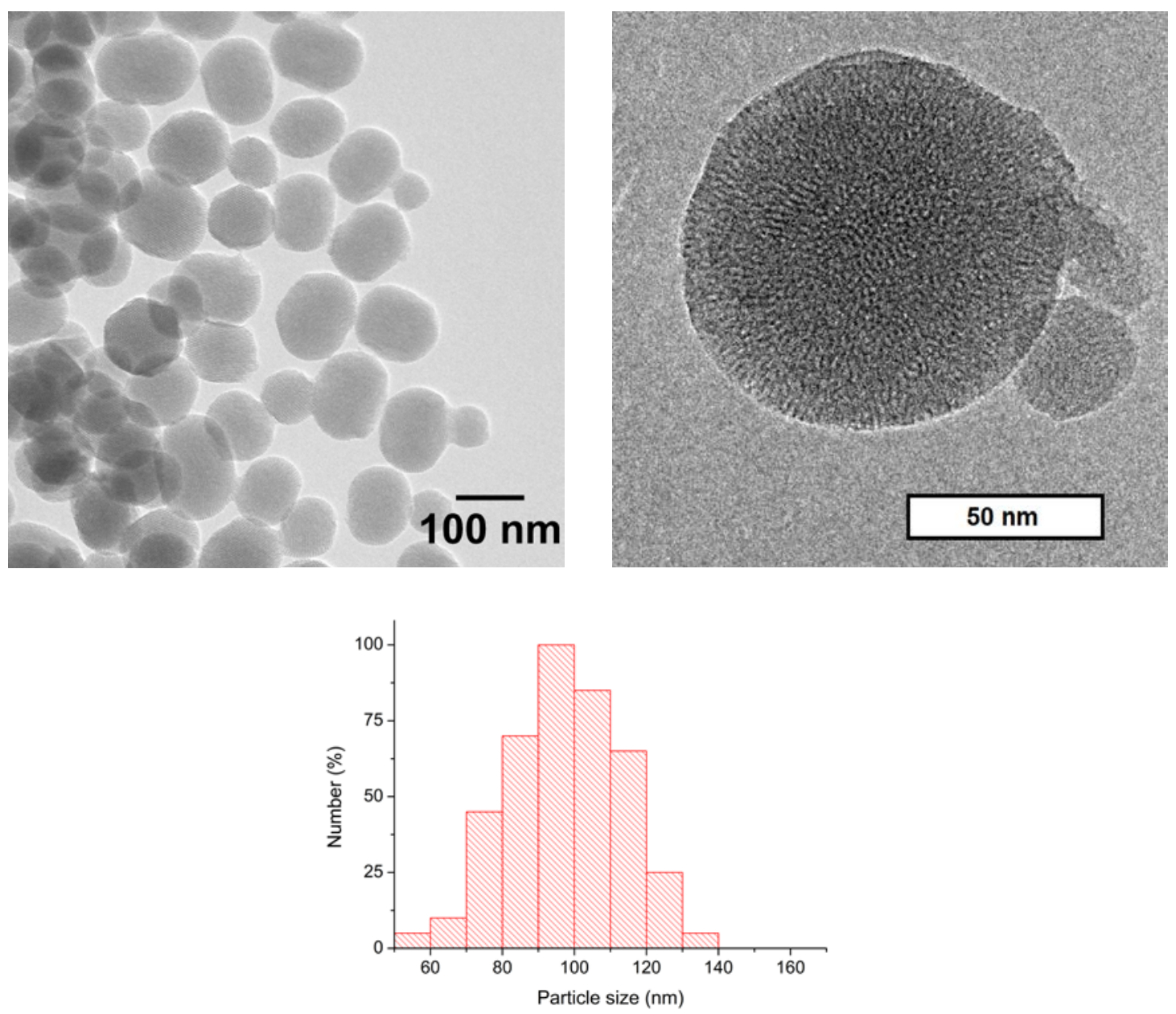

Figure 1S. TEM images and distribution of sizes of MS nanoparticles 

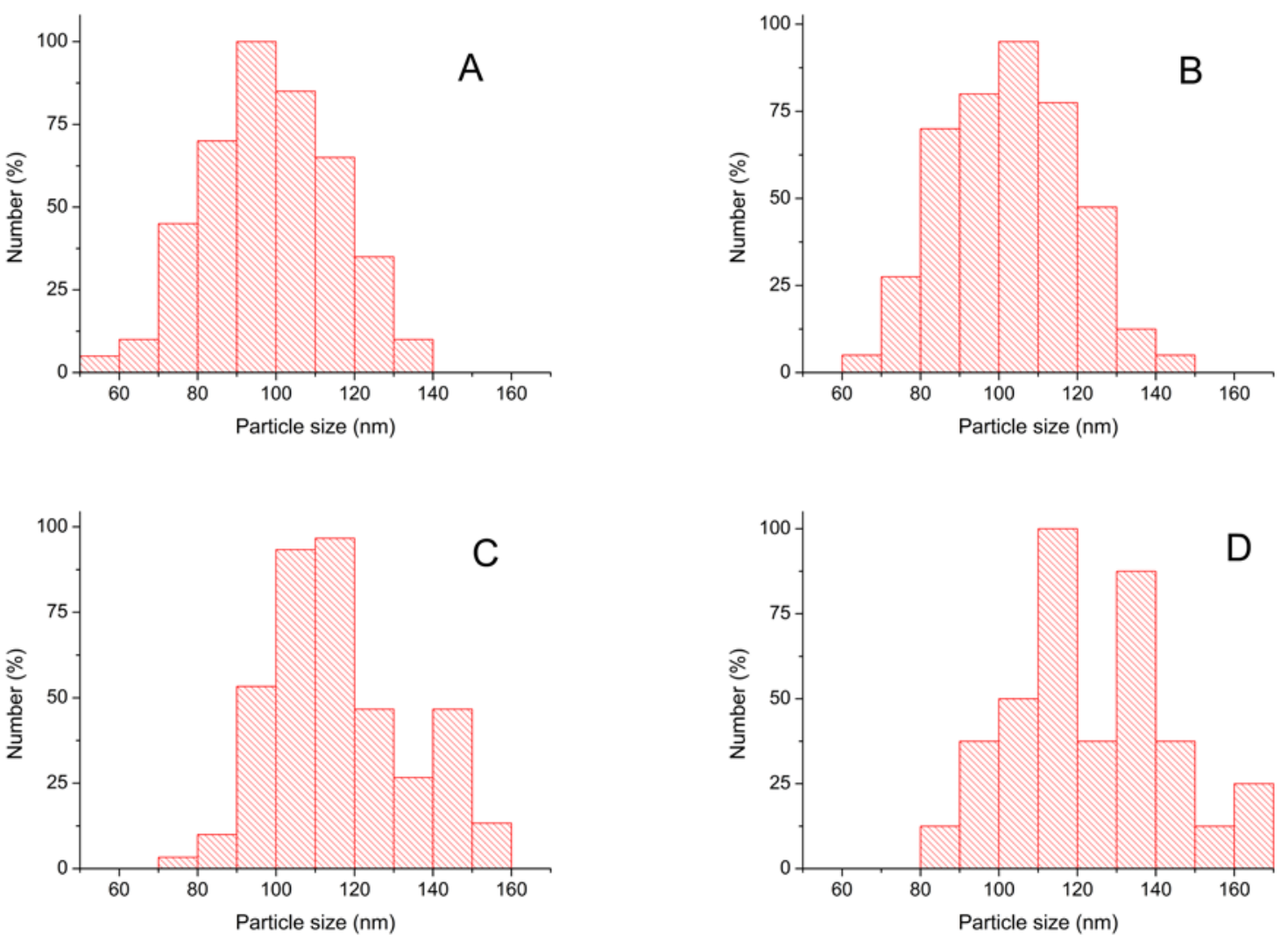

Figure 2S. Distribution of sizes of J1 (A), J2 (B), J3 (C) and J4 (D) nanoparticles 

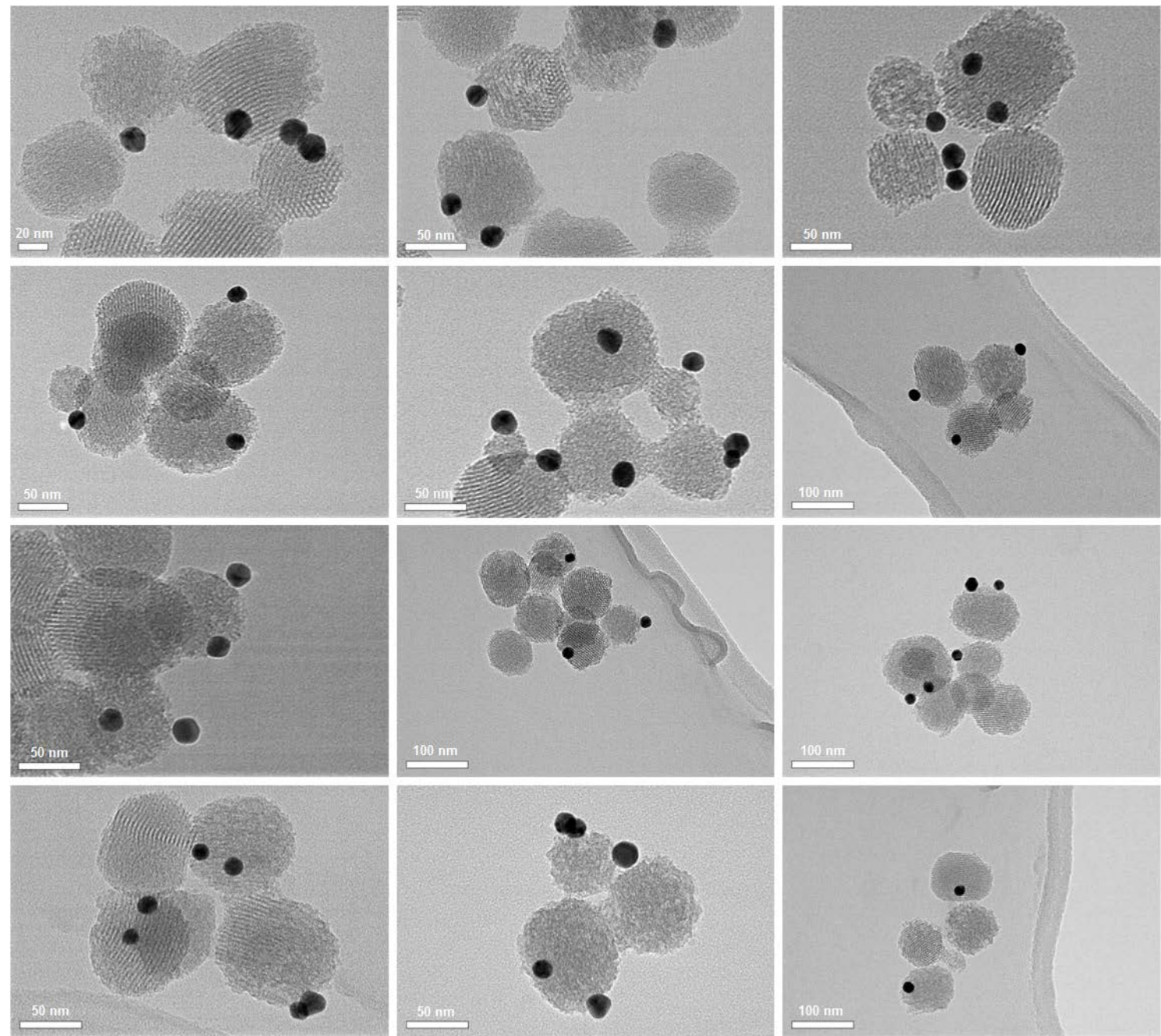

Figure 3S. TEM images of J2 nanoparticles. 